\title{
Fructosamine and glycated haemoglobin in the assessment of long term glycaemic control in diabetes
}

\author{
J P H Shield, K Poyser, L Hunt, C A Pennock
}

\begin{abstract}
Fructosamine and glycated haemoglobin were measured simultaneously in 147 children with diabetes. If glycated haemoglobin is considered as the 'gold standard' for long term glycaemic control, then fructosamine is a poor indicator of actual glycated haemoglobin values, with wide 95\% confidence (fiducial) limits. This shows that it is impossible to accurately predict glycated haemoglobin concentrations and therefore, by implication, longer term glycaemic control, from measurements of fructosamine. As the major studies on the prevention of microvascular complications in diabetes have used glycated haemoglobin levels to assess glycaemic control, it is suggested that this measurement should be used in all children with diabetes in preference to the measurement of fructosamine.

(Arch Dis Child 1994; 71: 443-445)
\end{abstract}

Many clinical chemistry laboratories are offering serum fructosamine determinations instead of glycated haemoglobin determinations for assessing diabetes control, the major consideration being cost. Fructosamine is a measure of the non-enzymatic glycation of proteins in blood, the major constituent of which is albumin. ${ }^{1}$ Glycated haemoglobin reflects integrated glycaemia over the preceding six to eight weeks, ${ }^{2}$ whereas fructosamine reflects glycaemic control over the previous two to three weeks. ${ }^{3}$ Several reports have compared these two methods of assessing glycaemic control in children. By using simple correlation analysis some have found relatively good correlations of 0.82 to 0.86 and have suggested that fructosamine is a reliable alternative to the determination of glycated haemoglobin. ${ }^{45}$ Other studies, however, have found that the two tests can produce different perceptions of glycaemic control. ${ }^{67}$ Nevertheless, many laboratories still offer fructosamine as the routine test for assessing glycaemic control in children with diabetes. Contact was made with the 11 clinical chemistry laboratories with a role in the analysis of glycaemic control of children with diabetes in the south west of England. All laboratories were asked whether fructosamine or glycated haemoglobin was used for the routine assessment of glycaemic control in their paediatric diabetes clinics: all responded. In two laboratories only fructosamine was measured; in a further two, both glycated haemoglobin and fructosamine were analysed. In seven laboratories, glycated haemoglobin was the preferred method.

The Diabetes Control and Complications Trial has now reported the impact of improved glycaemic control on long term microvascular disease in diabetes. Using glycated haemoglobin as one of the major determinants of control, it found that improvements in glycaemic control brought about major reductions in the mean risks of developing nephropathy, neuropathy, and retinopathy. ${ }^{8}$ As the 'gold standard', albeit imperfect due to the various methods of analysis used, glycated haemoglobin analysis is likely to become increasingly important for monitoring the effectiveness of intensified diabetic management. This study was designed to address the question of whether fructosamine analysis, measuring glycaemic control over the previous two to three weeks, is a reliable indication of glycated haemoglobin concentrations and, by implication, longer term control.

\section{Patients and methods}

Blood was collected from 147 children from a paediatric diabetes clinic for glycated haemoglobin and fructosamine analysis. The samples were taken from fingerpricks at the time of clinic visits. Fifty of the children were prepubertal (mean age 8.1 years). The other 97 children had a mean age of 14.6 years. Glycated haemoglobin was measured by a Corning electrophoretic electroendosmosis technique and fructosamine by measuring the change in absorbance resulting from the reduction of nitroblue tetrazolium on a Hitachi 717 random access analyser.

\section{STATISTICAL METHODS}

Fructosamine and glycated haemoglobin concentrations were logarithmically transformed (base $_{10}$ ) before statistical analysis to yield a better linear relation between them and to remove the positive skewness in the measurements. Taking glycated haemoglobin as the gold standard measurement, we were interested in examining the mean and range of measurements of fructosamine that would be obtained for a given concentration of glycated haemoglobin. We therefore fitted a regression line of the (log transformed) fructosamine on glycated haemoglobin, together with a $95 \%$ confidence interval for a predicted value. We then used inverse regression ${ }^{9}$ to determine values of glycated haemoglobin (together with $95 \%$ fiducial intervals) that would be 


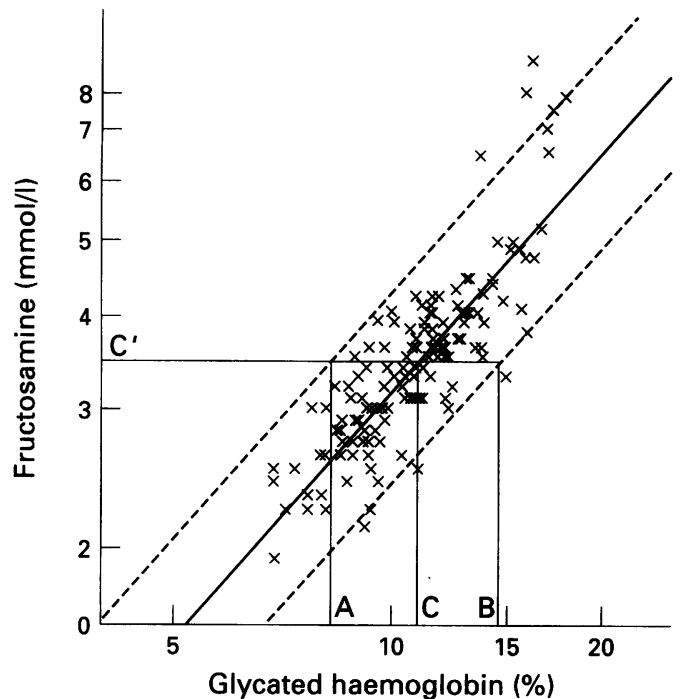

Plot of glycated haemoglobin against fructosamine showing line of regression and $95 \%$ confidence intervals for predicted value of glycated haemoglobin $\log _{10}$ scale used for both axes). $C^{\prime}=$ fructosamine, $3.5 \mathrm{mmoll} ; C=$ glycated haemoglobin, $11 \cdot 1 \% ; A=$ glycated haemoglobin, $8 \cdot 4 \%$, and $B=$ glycated haemoglobin, $14 \cdot 7 \%$.

compatible with individual fructosamine values of $2 \cdot 7,3 \cdot 2$, and $3.5 \mathrm{mmol} / 1$; these values are our laboratory's upper limit of good, middle value of moderate, and lower limit of bad control. The corresponding values used for glycated haemoglobin were $9.9,11$, and $12 \cdot 1 \%$ respectively.

\section{Results}

The figure shows the regression line and $95 \%$ confidence intervals for predicted values of (log) fructosamine. We expected a fructosamine concentration of $3.5 \mathrm{mmol} / 1\left(\mathrm{C}^{\prime}\right)$ to have been obtained from a glycated haemoglobin value of $11 \cdot 1 \%(\mathrm{C})$, but an individual value of $3.5 \mathrm{mmol} / 1$ could be compatible with glycated haemoglobin levels from $8 \cdot 4$ to $14 \cdot 7 \%$ (A to B respectively). Similarly, a fructosamine concentration of $3.2 \mathrm{mmol} / \mathrm{l}$ would be expected to have arisen from a glycated haemoglobin level of $10.2 \%$, but would be compatible with glycated haemoglobin values from $7 \cdot 7$ to $13.5 \%$. For a value of $2.7 \mathrm{mmol} / 1$, the corresponding values of glycated haemoglobin are $8 \cdot 7 \%$, and 6.5 to $11.5 \%$.

The sensitivity and specificity for predicting poor control in the determination of glycated haemoglobin greater than $12 \%$ using a fructosamine concentration greater or equal to 3.5 $\mathrm{mmol} / \mathrm{l}$ (our laboratory's lower limit for bad control) were respectively $88 \%$ and $68 \%$

Number of patients and agreement in clinical classification between fructosamine and glycated haemoglobin. Values in parentheses are the percentage of patients for a given fructosamine range in each of the glycated haemoglobin ranges

\begin{tabular}{lllll}
\hline \multicolumn{5}{l}{ Glycated haemoglobin } \\
\cline { 2 - 5 } Fructosamine & $\begin{array}{l}\text { Good } \\
(<10 \cdot 0 \%)\end{array}$ & $\begin{array}{l}\text { Moderate } \\
(10-12 \%)\end{array}$ & $\begin{array}{l}\text { Bad } \\
(>12 \cdot 0 \%)\end{array}$ & Total \\
\hline Good $(\leqslant 2 \cdot 7 \mathrm{mmol} / \mathrm{l})$ & $29(91)$ & $3(9)$ & 0 & $32(100)$ \\
Moderate $(2 \cdot 8-3 \cdot 4 \mathrm{mmo} / \mathrm{l})$ & $19(43)$ & $20(46)$ & $5(11)$ & $44(100)$ \\
Bad $(\geqslant 3 \cdot 5 \mathrm{mmol} / \mathrm{l})$ & $4(6)$ & $29(41)$ & $38(54)$ & $71(100)$ \\
Total & $52(35)$ & $52(35)$ & $43(29)$ & $147(100)$ \\
\hline
\end{tabular}

(table). The prevalence of poor control defined by glycated haemoglobin concentration in the study population was $29 \%$. The positive predictive value of fructosamine was $54 \%$ Furthermore, $44 \%$ of all the measurements reflecting good long term control by glycated haemoglobin analysis corresponded to a fructosamine concentration in either the bad or moderate control range. Fructosamine values other than $3.5 \mathrm{mmol} / \mathrm{l}$ were also used to define the lower limit for bad control to determine glycated haemoglobin values greater than $12 \%$, but all proved to have greater rates of error.

\section{Discussion}

Children are seen routinely in paediatric diabetes clinics every three to four months. Fructosamine only reflects blood glucose control over the previous two to three weeks, whereas glycated haemoglobin reflects control over the previous two months. Glycaemic control in children can vary significantly over a two week period due to factors such as intercurrent illness or holidays, thereby not reflecting average glycaemic control between clinic visits. Glycated haemoglobin values, however, are much less affected by these 'variations' in routine glycaemic control.

It is not surprising that the predictive value of fructosamine for poor control, compared with analysis by glycated haemoglobin, is only $54 \%$. An indication of poor glycaemic control from fructosamine determination might necessitate unnecessary changes in diabetes management simply because blood glucose concentrations in a two week period before a clinic visit were unusually poor. The broad range of the $95 \%$ confidence intervals makes the prediction of glycated haemoglobin from a fructosamine assay relatively meaningless. This is important as it is impractical and undesirable to see children routinely every three weeks. With much wider intervals between visits it seems hardly sufficient to rely on fructosamine measurements to make assumptions about longer term glycaemic control when there is such disparity between the two tests. The need to improve long term glycaemic control is now of paramount importance as three separate studies have suggested that this is a major predictor of neuropathy, retinopathy, and nephropathy using glycated haemoglobin as the yardstick for estimating control. ${ }^{10}$

Fructosamine does have its uses - for instance, in the monitoring of pregnant women with diabetes when frequent visits and estimation of glycaemic control are necessary. ${ }^{11}$ This is not the same situation as in the care of children with diabetes who are seen, at most, four times a year. We conclude that it is unsatisfactory to monitor fructosamine concentrations alone to assess long term glycaemic control between clinic visits. Not only is fructosamine of little help as it only tells us about glycaemic control in the two to three weeks before clinic visits, but we have also shown that it is inadequate for predicting the corresponding glycated haemoglobin. With the evidence 
accumulating as to the importance of long term glycaemic control, we must revert to the determination of glycated haemoglobin.

We thank Mrs J Jones and Mrs R Holton of the department of clinical chemistry in Bristol Royal Infirmary for their help with the laboratory analysis.

1 Johnson RN, Metcalf PA, Baker JR. Fructosamine: a new approach to the estimation of serum glycosylprotein. An index of diabetic control. Clin Chim Acta 1982; 127: 87-95.

2 Koenig RJ, Peterson CM, Jones RL, Saudek C, Lehrman $\mathrm{M}$, Cerami A. Correlation of glucose regulation and haemoglobin $\mathrm{A}_{\mathrm{lc}}$ in diabetes mellitus. N Engl f Med 1976; 295: 417-20.

3 Baker JR, O'Connor JP, Metcalf PA, Lawson MR, Johnson $\mathrm{RN}$. Clinical usefulness of estimation of serum fructosamine concentration as a screening test for diabetes melsamine concentration as a scre
litus. $B M \mathcal{F}$ 1983; 287: 863-7.

4 Cefalu WT, Mejia E, Puente GR, Fleishhacker D, Macaulay $\mathrm{K}$. Correlation of serum fructosamine activity in type 1 diabetic children. Am $\mathcal{F}$ Med Sci 1989; 297: 244-6.
5 Hindle EJ, Rostron GM, Clark SA, Gatt JA. Serum fructosamine and glycated haemoglobin measurements in diabetic control. Arch Dis Child 1986; 61: 113-7.

6 Watts GF, Macleod AF, Benn JJ, et al. Comparison of real-time use of glycosylated haemoglobin and plasma fructosamine in the diabetic clinic. Diabetic Med 1991; 8: 573-9.

7 Lee PDK, Sherman LD, O'Day MR, Rognerud CL, Ou C-N. Comparisons of home blood glucose testing and glycated protein measurements. Diabetes Res Clin Prac 1992; 16: 53-62.

8 The Diabetes Control and Complications Trial. The effect of intensive treatment of diabetes on the development and progression of long-term complications in insulin dependent diabetes mellitus. $N$ Engl 7 Med 1993; 329: 977-86.

9 Draper NR, Smith H. Applied regression analysis. 2nd Ed. Wiley series in probability and mathematical statistics. Chichester: Wiley, 1981: 47-51.

10 Shield JPH, Baum JD. Prevention of long-term complications of diabetes. Arch Dis Child 1994; 70: 258-9.

11 Parfitt VJ, Clark JDA, Turner GM, Hartog M. Use of fructosamine and glycated haemoglobin to verify self blood glucose monitoring data in diabetic pregnancy. Diabetic Med 1993; 10: 162-6. 